\title{
Prevalencia de anemia y estado nutricional de escolares del área periurbana de Sucre, Bolivia
}

\section{Anemia prevalence and nutritional status in children of a suburban area of Sucre, Bolivia}

\begin{abstract}
Background: There is limited data on the nutritional status and the prevalence of anemia among schoolchildren of Bolivia. This study aimed to determine the prevalence of anemia, iron deficiency and nutritional status by anthropometry in children of a suburban area in the Municipality of Sucre, Bolivia. Subjects and methods: A cross-sectional observational study was conducted involving all schoolchildren aged 6 to 10 years $(n=195)$ of a suburban school of Sucre-Bolivia ( $2750 \mathrm{~m}$ over sea level). We measured weight and height and calculated Z score for weight/age, height/age, and $\mathrm{BM} \mathrm{I/age} \mathrm{indices} \mathrm{using} \mathrm{WHO} \mathrm{standards.} \mathrm{Blood} \mathrm{samples} \mathrm{were}$ drawn to determine hemoglobin $(\mathrm{Hb})$ and mean corpuscular volume (MCV). Anemia was defined using cut-offs points adjusted for age and corrected for altitude ( $\mathrm{Hb}<13.4 \mathrm{~d} / \mathrm{dl}$ ). Results: The prevalence of malnutrition observed by $Z$ scores of height/age and weight/ age were $20 \%$ and $8,7 \%$, respectively, and mean BMI z score was $-0,07$. O f these children, $17.9 \%$ showed anemia (17.4\% iron deficiency anemia), 22.6\% iron deficiency without anemia and $19.5 \%$ depleted iron stores. Conclusions: The school age children from the suburban area of Sucre-Bolivia presented a mild prevalence of anemia and medium prevalence of stunting. Key words: anemia; iron deficiency, stunting, school age children.
\end{abstract}

\section{INTRODUCCIÓN}

El hierro es un oligoelemento mineral indispensable para el humano, participa en procesos biológicos, tales como el transporte y almacenamiento de oxígeno, y en la síntesis de hemoglobina (1). Además, es fundamental para el funcionamiento apropiado de numerosas enzimas, por lo que su deficiencia puede afectar múltiples funciones metabólicas incluida la respuesta inmunológica (2).

La anemia por deficiencia de hierro es una enfermedad nutricional de alta prevalencia en todo el mundo, especialmente en países en vías de desarrollo (3). La OMS estima que son anémicos alrededor de $39 \%$ de niños menores de 5 años, 48\% de niños de 5 a 14 años, $42 \%$ de todas las mujeres y $52 \%$ de las mujeres embarazadas de los países en desarrollo (3-4). En Bolivia, la deficiencia de hierro constituye el trastorno nutricional más común y la principal causa de anemia, con una prevalencia general de anemia del 56\% (5), alcanzando un $86,6 \%$ de niños entre 6 a 23 meses de edad (6) y $34.7 \%$ en escolares (7). Actualmente, Bolivia se encuentra entre los países con los porcentajes más elevados de desnutrición crónica (8), tres de cada diez niños menores de 5 años tienen talla baja para la edad, tasa nacional que enmascara
Melissa Miranda (1)

Manuel Olivares G (1) Jenny Durán-Pérez (2) Fernando Pizarro A. (1)

(1) Laboratorio de Micronutrientes, U nidad de N utrición Humana, Instituto de Nutrición y Tecnología de Alimentos (INTA), U niversidad de Chile. Santiago, Chile.

(2) Facultad de Bioquímica y Farmacia, U niversidad San Francisco Xavier de Chuquisaca, Sucre-Bolivia.

Dirigir la correspondencia a: Profesor

Fernando Pizarro A

Laboratorio de Micronutrientes, Unidad de Nutrición Humana Instituto de Nutrición y Tecnología de Alimentos (INTA), Dr. Fernando M onckeberg Barros

Universidad de Chile. Santiago, Chile. E-mail: fpizarro@inta.uchile.cl

Este trabajo fue recibido el 21 de Julio de 2015 y aceptado para ser publicado el 23 de Octubre de 2015.

marcadas inequidades, por ejemplo, el 41,8\% de los niños de la población más pobre, padece desnutrición crónica, versus $5.4 \%$ de los niños de la población más rica (9). Aún cuando en el último tiempo se han implementado Programas de Alimentación es escasa la información sobre la prevalencia de anemia y el estado nutricional en niños escolares de Bolivia tanto a nivel nacional como departamental.

Objetivo: Determinar la prevalencia de anemia, deficiencia de hierro y el estado nutricional por antropometría en niños escolares del área periurbana del Municipio de Sucre-Bolivia.

\section{SUJETOS Y MÉTODOS}

En agosto del 2010, en un estudio de tipo transversal -observacional se estudiaron a todos los escolares de 6 a 10 años ( $n=195)$, de una escuela del área periurbana de Sucre, Bolivia (2750 m.s.n.m.), población principalmente de origen quechua.

El equipo humano básico para la recolección de datos estuvo compuesta por bioquímicas de la Universidad de San Francisco Xavier de Chuquisaca, quienes se encargaron de la toma de muestras de sangre, un médico, responsable del 
examen clínico a los niños y una nutricionista para la colecta de los datos antropométricos.

Consideraciones éticas: El presente estudio fue aprobado por el Comité de Ética del Instituto de Nutrición y Tecnología de Alimentos (INTA) de la Universidad de Chile y por las autoridades de salud y educación del Municipio de Sucre. Los padres o apoderados de los sujetos recibieron información detallada sobre el estudio y los que aceptaron participar firmaron un consentimiento informado, obteniéndose además el asentimiento por parte de los niños.

Determinaciones bioquímicas: Después de un ayuno de 8 horas se obtuvo una muestra de $3 \mathrm{ml}$ de sangre por punción venosa cubital para determinar hemoglobina $(\mathrm{Hb})$, volumen corpuscular medio (VCM), ferritina sérica (FS) y proteína $C$ reactiva (PCR). La hemoglobina y $V C M$ se midieron un contador electrónico de células (Horiba ABX Micros 60) en el Laboratorio de la Facultad de Bioquímica de la Universidad San Francisco Xavier de Chuquisaca, Sucre-Bolivia. En el Laboratorio de Micronutrientes del Instituto de Nutrición y Tecnología de Alimentos de la Universidad de Chile, se determinó ferritina sérica por ELISA (10) y proteína $C$ reactiva (Turbox. Orion diagnostica. Espoo. Finlandia). Anemia se definió como $\mathrm{Hb}<134 \mathrm{~g} / \mathrm{l}$; anemia por deficiencia de hierro $\mathrm{Hb}$ alterada más $\mathrm{VCM}<80 \mathrm{fL}$ y/o $\mathrm{FS}<30 \mathrm{ug} / \mathrm{L}$; deficiencia de hierro sin anemia como $\mathrm{Hb}$ normal y VCM y FS alterados, y depósitos depletados de hierro como solo FS alterada. Para definir el estatus de hierro se utilizaron puntos de cortes establecidos por la OMS (4) y los valores de hemoglobina fueron corregidos por altitud (11). Dado que la ferritina sérica es una proteína de fase aguda que aumenta con la inflamación y la infección, uno de los métodos utilizados para obviar este factor en aquellas poblaciones con alta prevalencia de infección utiliza un punto de corte de $30 \mathrm{ug} / \mathrm{L}$ de FS (12).

M ediciones antropométricas: Los datos antropométricos de peso y talla fueron obtenidos de acuerdo a las Guías para Medir Indicadores Antropométricos del proyecto FANTA de la Academia de Desarrollo Educacional de USA (13). Para la medición del peso corporal los niños se presentaron con el mínimo de ropa y sin zapatos; se utilizó una balanza digital (Camry, precisión de 10 g) y la talla se midió con un estadiómetro portátil fijo a la pared (Seca-206, precisión de 0,1 $\mathrm{cm})$. Los datos fueron registrados en la ficha de seguimiento del niño.

Evaluación del estado nutricional: Para la evaluación del estado nutricional se calculó puntaje Z usando los estándares de crecimiento WHO 2007 para niños de 5 a 19 años (14). Las fechas de nacimiento de los niños proporcionadas por las listas de matrícula fueron utilizadas para determinar la edad cronológica en meses. El retraso del crecimiento y el bajo peso se definió como puntaje $Z$ menor a -2 DE para talla/ edad y peso/ edad, respectivamente (14). Se calculó el índice de masa corporal (IMCZ) y fue clasificado para determinar el estado nutricional en cuatro intervalos: $1=$ bajo peso (IMCZ $\leq$ $-1), 2=$ normal $(I M C Z>-1 \leq 1), 3=$ sobrepeso $(I M C Z>1 \leq 2)$, 4=obesidad (IMCZ > 2).

Análisis estadístico: Los resultados fueron analizados con estadística descriptiva, reportando los valores de concentración como promedio, desviación estándar o porcentajes.

\section{RESULTADOS}

Participaron en este estudio 107 niños y 88 niñas de 6 a 10 años, con un promedio de edad de 8,3 años. La tabla 1 muestra el estado de nutrición de los niños estudiados, destaca el bajo promedio de $Z$ de la talla para la edad y $Z$ de peso para la edad tanto en niños como niñas, sin diferencias por género. El promedio de $Z$ de IMC de los niños se aproxima a cero. El 17,7 y $22,7 \%$ de niños y niñas tiene talla baja. La figura 1 muestra que un 12,3\% de los niños tienen bajo peso, $8,2 \%$ sobrepeso y sólo $2 \%$ de los niños presentan obesidad. Los promedios de HB, VCM y FS de todos los niños fueron $141 \pm 8 \mathrm{~g} / \mathrm{L}, 81 \pm 2 \mathrm{fL}$ y $31 \pm 15 \mathrm{ug} / \mathrm{L}$ respectivamente, sin diferencias en los promedios por género. La figura 2 muestra que $17,4 \%$ de los niños presentaban anemia por deficiencia de hierro, 22,6\% deficiencia de hierro sin anemia y 19,5\% un estado de los depósitos de hierro depletados. En total 59,5\% de los niños tenían alterado su estado nutricional de hierro. No se identificaron casos de anemia severa $(\mathrm{Hb}<8.3 \mathrm{~g} / \mathrm{dL})$, y que $96 \%$ de los escolares tenían PCR alterada (>5 mg/L).

\section{DISCUSIÓN}

En este estudio se evaluó la prevalencia de anemia, el estado nutricional de hierro y el estado nutricional determinado por medidas antropométricas de escolares de una escuela del área peri-urbana de Sucre-Bolivia. Nuestros resultados revelaron una prevalencia de deficiencia de hierro significativamente menor a las publicadas por San Miguel y cols. en escolares de una zona periférica de La Paz (edades, 6-13 años), ellos encontraron que un $52,4 \%$ presentaban anemia y $95,2 \%$ tenían ferritinas bajo lo normal (15), comparado con un $17,4 \%$ de anemia y $19,5 \%$ de escolares con depósitos depletados en este estudio. Según la última Encuesta Nacional de Salud realizada en Bolivia el año 2008 (ENDSA-2008)(16), $61.3 \%$ de los niños menores de 6 años presentan algún grado de anemia, 10 puntos porcentuales más de lo estimado en la ENDSA-2003 (17). El año 2009, se informó que la pre-

TABLA 1

Estado nutricional de escolares de Sucre-Bolivia

\begin{tabular}{lccc}
\hline & $\begin{array}{c}\text { Niños } \\
(\mathbf{n = 1 0 7 )}\end{array}$ & $\begin{array}{c}\text { Niñas } \\
(\mathbf{n = 8 8 )}\end{array}$ & $\begin{array}{c}\text { Total } \\
(\mathbf{n = 1 9 5 )}\end{array}$ \\
\hline Edad (años)1 & $8,2 \pm 1,3$ & $8,4 \pm 1,3$ & $8,3 \pm 1,3$ \\
Z IMC1 & $0,02 \pm 0,8$ & $-0,17 \pm 1,0$ & $-0,07 \pm 0,9$ \\
Z Talla para la edad & $-1,3 \pm 0,8$ & $-1,4 \pm 1,0$ & $-1,4 \pm 0,9$ \\
Z Peso para la edad & $-0,8 \pm 0,9$ & $-0,9 \pm 0,9$ & $-0,9 \pm 0,8$ \\
Indicador Talla/edad (\%) & 17,7 & 22,7 & 20,0 \\
Baja talla & 17,7 & 2 \\
\hline
\end{tabular}

1Promedios y desviación estándar 
valencia de anemia en una muestra representativa de escolares beneficiarios del programa Complemento Alimentario Escolar del departamento de La Paz fue 22, 31 y 15\% para las provincias de Camacho, Ingaví y Murillo respectivamente (18). Estas prevalencias son comparables a las encontradas en nuestro estudio. La OMS estima que es un problema leve de salud pública cuando la prevalencia de anemia fluctúa entre $>5 \%$ y $>20 \%$ y es moderada entre 20 y $<40 \%$ (4). Bolivia, de acuerdo a las características de su dieta presentaría una absorción de hierro de 5 a 10\% según criterios establecidos por la FAO/OMS (19). Por lo tanto, este factor es probable- mente la causa principal de la alta prevalencia de anemia encontrada en esta población.

Por otra parte, las prevalencias de desnutrición observadas mediante los puntajes Z de talla/edad (20\%) de los niños estuvieron cercanas a los valores nacionales (27\%) reportados en la ENDSA-2008 (16). La OMS clasifica como un problema moderado de retraso de talla a prevalencias entre $20 \mathrm{y}$ $<30 \%$ (20). Como se evidenció con los resultados obtenidos en este estudio al igual que en la ENDSA-2008, el retardo del crecimiento sigue siendo el problema principal desde el punto de vista antropométrico.

FIGURA 1.

Estado nutricional de escolares de una zona suburbana de Sucre, Bolivia.

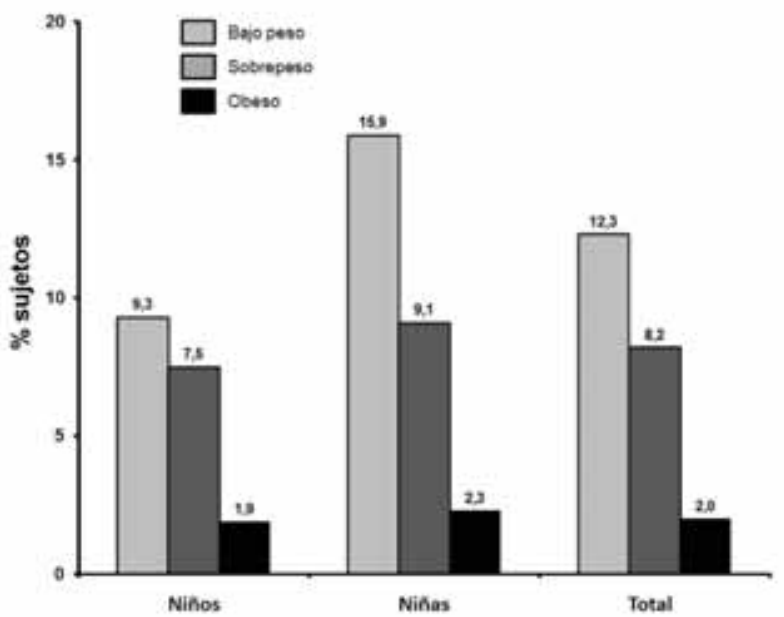

FIGURA 2

Estado de nutrición de hierro de escolares de una zona suburbana de Sucre-Bolivia.

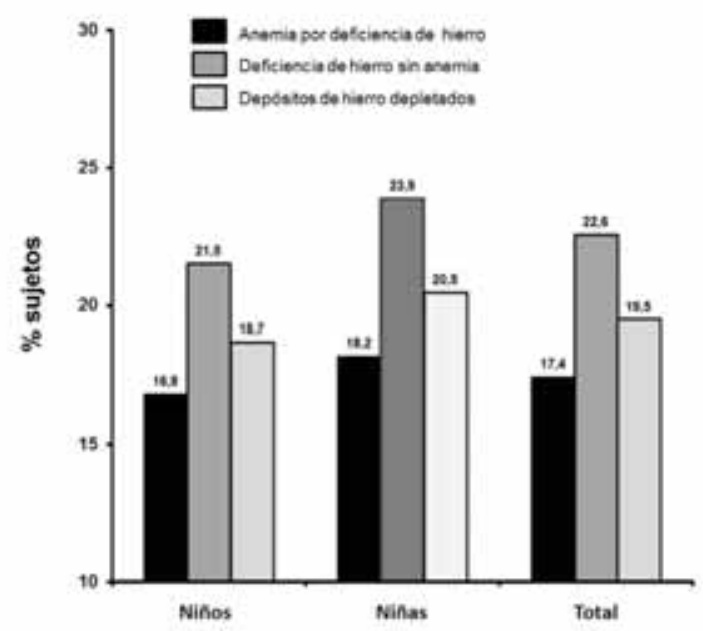


En un estudio realizado por Weisstaub y cols. (21) en escolares de la misma de edad de Tocopaya, Bolivia (3100 m.s.n.m.) encontró resultados muy dispares comparados con este estudio, en una muestra de 112 niños reportó 41\% de talla para la edad $<$ a -2 Z. Bolivia se encuentra entre los países de Latinoamérica con los porcentajes más elevados de desnutrición, (27\%) (22), además lamentablemente debido a las marcadas disparidades de la región las probabilidades de presentar una baja talla en poblaciones como la del presente estudio son más elevadas que quienes viven en zonas urbanas. El detrimento de la población de las zonas rurales con respecto a las urbanas en América Latina ha sido reportado por otros autores (23-24).

Los resultados de este estudio justifican la continuación de programas de Alimentación complementaria y fortificación de los alimentos con hierro para la prevención de la anemia ferropriva, especialmente en escolares de bajo nivel socioeconómico.

\section{RESUMEN}

Introducción: Existen escasos datos sobre el estado nutricional y la prevalencia de la anemia entre los escolares de Bolivia. Este estudio tuvo como objetivo determinar la prevalencia de anemia, deficiencia de hierro y el estado nutricional por antropometría en niños de una zona suburbana de la Municipalidad de Sucre, Bolivia. Sujetos y métodos: Se realizó un estudio observacional transversal en que participaron todos los escolares de 6 a 10 años $(n=195)$ de una escuela suburbana de Sucre-Bolivia (2750 msnm). Se midió el peso y la talla y se calculó puntaje Z peso/edad, talla/edad, e IMC utilizando tablas de la OMS. Se extrajo una muestra de sangre para determinar hemoglobina, volumen corpuscular medio y ferritina sérica. La anemia se definió utilizando un punto de corte por edad y corregidos por la altitud $(\mathrm{Hb}<13.4 \mathrm{~d} / \mathrm{dl}$ ). Resultados: La prevalencia de malnutrición observado por las puntuaciones Z de talla/edad y peso/edad fueron de $20 \%$ y $9 \%$, respectivamente, y la media del z de IMC fue $-0,07$. De estos niños, 17, 9\% presentó anemia (17,4 anemia por deficiencia de hierro), 22,6\% deficiencia de hierro sin anemia y 19,5\% depósitos de hierro depletados. Conclusiones: Los niños en edad escolar de la zona suburbana de Sucre-Bolivia presentaron una prevalencia leve de anemia, y una moderada prevalencia de retraso del crecimiento.

Palabras clave: anemia; deficiencia de hierro, retraso de talla, escolares.

\section{BIBLIOGRAFIA}

1. Olivares $M$, Arredondo $M$, Pizarro F. Hierro. En: Gil A, ed. Tratado de nutrición. $2^{a}$ ed. Madrid, Editorial Médica Panamericana; 2010. v. 1, p. 668-86.

2. Beard $J$, Dawson $H$, Piñero DJ. Iron metabolism: a compressive review. Nutr Rev 1996;54:295-317.

3. World Health Organization. Worldwide prevalence of anemia, 1993-2005: WHO global database on anemia. Geneva: WHO, 2008.

4. WHO/UNICEF/UNU. Iron Deficiency Anemia Assessment, Prevention, and Control. World Health Organization, Geneva; 2001.

5. Organismo Regional Andino de Salud - Convenio Hipólito Unanue. Plan Andino de Erradicación de la Malnutrición Infantil: Análisis de la Situación de Malnutrición Infantil en la Subregión Andina. Lima, Perú; 2006.

6. Urquidi B., Cinthya, Trujillo N, Mejía H. et al. Prevalencia de anemia en niños de 6 a 24 meses de edad de tres centros de salud de la ciudad de La Paz. Rev Bol Ped. 2006, 45:153-56.

7. Alurralde L. Perfil Nutricional de Bolivia. En: Alimentación y Nutrición en la última década del siglo XX. FAO, Roma; 2001.

8. UNICEF. Prevalencia de la Desnutrición Crónica (retraso del crecimiento) en 22 países de ALC - Promedios Nacionales. 2003.

9. OPS: Programa Desnutrición Cero de Bolivia: objeto de estudio e intervención desde el modelo conceptual de salud internacional. Organización Panamericana de la Salud, Bolivia; 2008.

10. Flowers CA, Kuizon $M$, Beard JL, Skikne BS, Covell AM, Cook JD. A serum ferritin assay for prevalence studies of iron deficiency. Am J Hematol. 1986;23:141-51.

11. Dirren $\mathrm{H}$, Logman $M \mathrm{H}$, Barclay DV, Freire WB. Altitude correction for hemoglobin. Eur J Clin Nutr. 1994;48:62532.

12. Joint World Health Organization/Centers for Disease Control and Prevention Technical Consultation on the Assessment of Iron Status at the Population Level. Geneva, Switzerland, 2004:p 9.

13. Cogill B, FANTA Project. Anthropometric Indicators Measurement Guide. Food and Nutrition Technical Assistance Project, Academy for Educational Development. Washington; 2003.

14. de Onis $M$, Onyango AW, Borghi E, Siyam A, Nishida $C$, Siekmann J. Development of a WHO growth reference for school-aged children and adolescents. Bull WHO 2007; 85:661-8.

15. San Miguel JL, Muñoz M, Urteaga N, Espejo E. Deficiencia de hierro y anemia en escolares residentes de gran altitud: asociación con infección. Cuad Hosp Clin. 2014; 55:24-33.

16. Ministerio de Salud y Deportes, Instituto Nacional de Estadística. Encuesta Nacional de Demografía y Salud ENDSA-2008. Ministerio de Salud y Deportes, La Paz, Bolivia, 2009.

17. Ministerio de Salud y Deportes, Instituto Nacional de Estadística. Encuesta Nacional de Demografía y Salud ENDSA-2003. Ministerio de Salud y Deportes, La Paz, Bolivia, 2004.

18. San Miguel JL. Complemento Alimentario Escolar: una estrategia contra la anemia nutricional. Asociación Cuna, La Paz, Bolivia, 2009.

19. FAO/WHO. Vitamin and Mineral Requirements. WHO, Geneva, 2004.

20. WHO. WHO Global Database on Child Growth and Malnutrition. World Health Organization, Geneva, 1997.

21. Weisstaub $G$, Bustos $M$, Olivares $M$, Durán-Castilllo $C$, Araya $M$. Situación nutricional de hierro, cobre y zinc en escolares de Tacopaya Bolivia. Rev Soc Bol Pediatr. 2004; 43(2): 77-80.

22. FAO. Perfiles Nutricionales por países. Bolivia. Organización de las Naciones Unidas para la Agricultura y la Alimentación (FAO), Roma, 2001.

23. Gueri $M$. Deficiencia de hierro en América Latina y el Caribe. En: Tercer Taller regional sobre deficiencia de vitamina A y otros micronutrientes en América Latina y el Caribe. Recife; Brasil, 1993; p 45-6.

24. Instituto de Nutrición de Centro América y Panamá (INCAP), Organismo Panamericano de la Salud (OPS). Encuesta Nacional de Micronutrientes, 1995; Guatemala. 1996. 